\title{
Perfil epidemiológico e caracterização das lesões em vítimas de homicídios: revisão narrativa
}

\author{
Epidemiological profile and characterization of injuries in homicide victims: narrative review
}

Perfil epidemiológico y caracterización de lesiones en víctimas de homicídio: revisión narrativa

Jonas Campos Cruz ${ }^{1 *}$, Beatriz Rodrigues de Freitas ${ }^{1}$, Larissa Eustáquia Passos Silva de Souza ${ }^{1}$, Patrick Alexandre dos Santos Oliveira ${ }^{1}$, Ruth Nathalia Cantaluppi Escobar ${ }^{1}$, Victor Fernando Bogado Argüello ${ }^{1}$, Vinícius Silva Belo ${ }^{1}$, Lucas Henrique Oliveira Amaral ${ }^{2}$, João Paulo Fonseca Nunes $^{2}$, Andressa Vinha Zanuncio ${ }^{1,2}$.

\section{RESUMO}

Objetivo: Organizar os estudos médico-legais nacionais e internacionais sobre a epidemiologia e caracterização das lesões em vítimas de homicídios, com o intuito de conhecer os dados epidemiológicos já registrados sobre o tema. Revisão bibliográfica: Indivíduos do sexo masculino de 20 a 40 anos foram a maioria das vítimas de homicídios. Condições socioeconômicas são um importante fator de risco para os homicídios. Estudos brasileiros constataram que 76,4\% dos indivíduos assassinados eram economicamente ativos e $78,1 \%$ possuíam o segundo grau de escolaridade completo. A maioria das vítimas residiam em áreas urbanas. Existe relação entre áreas de baixa qualidade de vida e violência urbana. Tratando-se das regiões anatômicas mais atingidas nos casos de homicídio por arma de fogo, houve destaque na região da cabeça, pescoço e peito. A análise dos achados toxicológicos das vítimas de constataram que as substâncias mais prevalentes foram álcool, seguido de narcóticos, cannabis e opiáceos. As características das lesões variam em cada estudo, entretanto também apresentam características que podem ser generalizadas. Considerações finais: Os números deste estudo podem servir de referência para a detecção e enfrentamento de questões de segurança pública. Foi dada como indiscutível a relevância de estudos deste cunho na atual realidade nacional.

Palavras-chave: Autópsia, Medicina forense, Homicídio, Armas.

\begin{abstract}
Objective: To organize national and international medico-legal studies on the epidemiology and characterization of injuries in homicide victims, in order to know the epidemiological data already registered on the subject. Bibliographic review: Male individuals aged 20 to 40 years were the majority of homicide victims. Socioeconomic conditions are an important risk factor for homicides. Brazilian studies found that $76.4 \%$ of the murdered individuals were economically active and $78.1 \%$ had completed high school. Most victims resided in urban areas. There is a relationship between areas of low quality of life and urban violence. As these are the most affected anatomical regions in cases of homicide by firearms, the head, neck and chest region stood out. The analysis of the toxicological findings of the victims found that the most prevalent substances were alcohol, followed by narcotics, cannabis and opiates. The characteristics of the lesions vary in each study, however they also have characteristics that can be generalized. Final considerations: The numbers in this study can serve as a reference for detecting and dealing with public safety issues. The relevance of studies of this nature in the current national reality was considered indisputable.
\end{abstract}

Keywords: Autopsy, Forensic medicine, Homicide, Weapons.

${ }^{1}$ Universidade Federal de São João del-Rei (UFSJ), Divinópolis - MG.

*E-mail: jonascamposcruz@gmail.com

${ }^{2}$ Polícia Civil de Minas Gerais, Divinópolis - MG. 


\section{RESUMEN}

Objetivo: Organizar estudios médico-legales nacionales e internacionales sobre epidemiología y caracterización de lesiones en víctimas de homicidio, a fin de conocer los datos epidemiológicos ya registrados sobre el tema. Revisión bibliográfica: Los hombres de 20 a 40 años fueron la mayoría de las víctimas de homicidio. Las condiciones socioeconómicas son un factor de riesgo importante para los homicidios. Estudios brasileños encontraron que el $76,4 \%$ de las personas asesinadas eran económicamente activas y el $78,1 \%$ había completado la escuela secundaria. La mayoría de las víctimas residían en áreas urbanas. Existe una relación entre áreas de baja calidad de vida y violencia urbana. Como estas son las regiones anatómicas más afectadas en casos de homicidio por arma de fuego, se destacó la región de la cabeza, el cuello y el pecho. El análisis de los hallazgos toxicológicos de las víctimas encontró que las sustancias más prevalentes fueron el alcohol, seguido de los narcóticos, el cannabis y los opiáceos. Las características de las lesiones varían en cada estudio, sin embargo, también tienen características que se pueden generalizar. Consideraciones finales: Los números de este estudio pueden servir como referencia para detectar y tratar problemas de seguridad pública. Se consideró indiscutible la relevancia de estudios de esta naturaleza en la realidad nacional actual.

Palabras clave: Autopsia, Medicina forense, Homicidio, Armas.

\section{INTRODUÇÃO}

O homicídio é uma das cinco categorias de mortes por causas externas da Classificação Estatística Internacional de Doenças e Problemas Relacionados à Saúde, em sua $10^{\underline{a}}$ Revisão (CID-10), correspondendo a uma importante parcela de óbitos em todos os países do mundo, especialmente na população masculina brasileira. Esta causa mortis representa o extremo da violência em uma sociedade e está sempre relacionada a graves problemas de ordem política e socioeconômica (TAVARES R, et al., 2016).

O Brasil tem a sexta maior população do mundo: 211 milhões de pessoas sendo $25 \%$ dela composta por menores de 18 anos (OLIVEIRA AS, 2019). Com queda da renda per capital nacional nos últimos anos, de 9.001,23 dólares em 2018, o país tem um dos mais altos índices de desigualdade do mundo: na última escala do coeficiente de GINI, o país entre se encontrou entre os dez países mais desiguais do mundo (SANTOS JAF, et al, 2018).

Com estes alarmantes níveis de desigualdade social, a violência vem se tornando um grande problema de saúde no pública no brasil. Desde os anos 30, doenças infecciosas vêm caindo na contagem de mortes enquanto as mortes causadas por violência mão aumentando cada vez mais. Em 2007, 12,5\% de todas as mortes em território nacional foram causadas por violência, em sua maioria, as vítimas eram homens jovens (BARRETO ML, et al., 2011).

Os custos sociais e econômicos da violência no Brasil são muito altos - o medo, os ferimentos físicos e os problemas orgânicos e psicológicos de saúde (ANDRADE LA, et al., 2012) têm impactos profundos na qualidade de vida dos cidadãos. Primeiramente, se tratando dos gastos em políticas sociais, no serviço de saúde e segurança públicas (policias e serviços de encarceramento), que são profundamente expressos no produto interno bruno (PIB). Além da relevante importância epidemiológica devido ao encerramento da vida de pessoas em plena atividade laboral, se trata de um evento multifatorial, complexo e com repercussão não somente sobre a qualidade, mas também sobre a expectativa de vida dos cidadãos (BORDONI PHC, et al., 2017).

Determinar a causa mortis e a sua origem com o estudo médico legal é essencial em uma investigação forense, o que permite conhecer a epidemiologia de eventos em geral e, dessa forma, instituir medidas que tragam a diminuição de mortes e acidentes em uma sociedade. Dialogar sobre a gravidade e os principais fatores relacionados aos homicídios tem utilidade que vai além da identificação de seus determinantes, sendo eficientes também para adequação de planejamentos, implementação e avaliação de ações ou programas internos de prevenção de violência (HAMDOUND M, et al., 2017). Refinar a análise das possíveis associações entre condições de saúde e vida são necessárias para a elaboração de indicadores sociais (de SOUZA ER, et al., 2018). 
Para que se possa entender as cenas e as pessoas envolvidas nos atos de homicídio pelo mundo, é preciso mapear o percurso da intervenção feita pelo governo. O que nos permite observar a estratégia adotada em vários países, de métodos mais punitivos e repressivos de controle dos cidadãos, na busca pelo maior controle da violência (ANTÃO KL, et al., 2019). Por outro lado, também existem países que adotam outras atitudes na busca pela diminuição da violência, com políticas sociais com fundamentos preventivos. Atuando nas causas determinantes do crime (TOYGAR M, et al., 2013).

Tratando-se de estudos de natureza médico-legal e epidemiológica, poucos trabalhos caracterizando as particularidades dos homicídios são feitos no Brasil, estes se limitando às regiões nordeste e sudeste. Assim, faz-se de muita importância a compreensão dos fatores relacionados ao homicídio - que não só ajudam a estabelecer seus determinantes, mas como também a planejar, implementar e avaliar adequadamente as ações ou planos de prevenção à violência por homicídio, e também a violência de modo geral (ORELLANA JDY, et al., 2017).

Este trabalho tem como objetivo organizar os estudos médico-legais nacionais e internacionais sobre a epidemiologia e caracterização das lesões em vítimas de homicídios, com o intuito de conhecer os dados epidemiológicos já registrados sobre o tema.

\section{REVISÃO BIBLIOGRÁFICA}

\section{O perfil epidemiológico das vítimas de homicídio}

\section{Cor, escolaridade e fatores socioeconômicos}

Os homicídios têm um forte significado social, pois além de tirarem a vida de cidadãos, também são reflexos dos problemas enfrentados pela nossa sociedade, como as desigualdades, a impenitência e a corrupção (HE M, et al., 2015; TAVARES R, et al., 2016).

Três artigos desta revisão sistemática avaliaram a situação da cor e da etnia dos periciados, o que indica a demanda para registro dessa característica em estudos deste tipo, pois a associação da cor do indivíduo pode interferir nos dados epidemiológicos. No geral, a maioria das vítimas de homicídios tem menos que 35 anos, pertencem a baixos estratos sociais e econômicos e tem baixa escolaridade. A cor e a etnia das vítimas tendem a variar com cada país e região (GARCÍA HI, et al., 2012; CLARK C, et al., 2017; MATZOPOULOS R, et al., 2015).

Em Medellín, na Colômbia, a caracterização dos homicídios ocorridos durante o período de 30 anos, entre 1979 e 2008, mostra que a concentração do perfil das vítimas é maior em homens jovens com idade inferior a 35 anos. A maioria das vítimas também era pertencente a baixos estratos socioeconômicos e residentes de favelas da cidade, onde há prevalência de baixa escolaridade. Essa vitimologia sugeriu aos autores que as condições socioeconômicas representam um importante fator de risco para os homicídios, uma vez que em praticamente todos os casos analisados, as motivações pessoais criminais giraram em torno de ajustes de contas, assaltos e brigas. Na Colômbia, de forma semelhante ao que ocorre no Brasil, pelo menos 8 em cada 10 homicídios estão associados com agressividade, intolerância e forma inadequada de resoluções conflitais (GARCíA HI, et al., 2012).

O estudo descritivo retrospectivo na África do Sul aborda as características mais comuns dos periciados e suas relações com mortes por lesão através de informações obtidas pelo levantamento de relatórios policiais e hospitalares do serviço de patologia forense por todo o país no ano de 2009 (CLARK C, et al., 2017). Diferentemente do que ocorre na realidade brasileira, na África do Sul a prevalência de homicídios em pessoas de cor preta e com condições econômicas precárias não foi tão discrepante quanto à dos indivíduos asiáticos, brancos, e os classificados como "coloured" (mestiços, indivíduos com características físicas herdadas de mais de uma etnia). Tais indivíduos, geralmente, são pessoas com condições econômicas semelhantes ou melhores do que as dos indivíduos negros. Foi registrado que nas regiões metropolitanas da África os homicídios de "não negros" por cada 10 mil habitantes são, inclusive, maiores do que a mesma taxa da população negra (MATZOPOULOS R, et al., 2015). 
Apenas 2 trabalhos brasileiros nessa revisão sistemática mencionaram a questão étnica e socioeconômica como dado epidemiológico dos periciados registrados pelo estudo. Os dois são estudos retrospectivos realizados em Belo Horizonte, capital do estado de Minas Gerais, que buscaram estratificar os óbitos ocorridos, respectivamente, por trauma abdominal e trauma nos membros, com os dados do Instituto Médico Legal de Belo Horizonte no período entre 2006 a 2011. Nestes estudos havia suspeita de homicídio pelo menos na cerca de metade dos casos (BORDONI PHC, et al., 2017; HELENA P, et al., 2017).

No primeiro foram avaliados 1888 laudos de óbitos ocorridos por trauma abdominal, número que corresponde à $5,2 \%$ do total de óbitos atendidos pelo Instituto Médico Legal (IML) de Belo Horizonte durante esse período. Nele, foi registrado que a maior parte dos laudos caracterizava indivíduos de cor morena, associando essa característica física com o estado civil solteiro e ao sexo masculino. Em outro trabalho brasileiro, foi feita uma busca pela observação de óbitos afetados por traumas de membros, estabelecendo uma estratificação dos traumas pelos indivíduos. O estudo, que avaliou 128 casos de lesões por traumas nos membros dos cadáveres de vítimas de homicídios, constata que a maioria dos indivíduos eram economicamente ativos $(76,4 \%$ dos casos), sendo que $4,7 \%$ deles eram aposentados, e, em relação ao nível educacional, 78,1\% possuíam o segundo grau de escolaridade completo (BORDONI PHC, et al., 2017; HELENA P, et al., 2017).

É importante salientar que as análises médico-legais que avaliam as situações de etnia das vítimas de homicídios nas necropsias são importantes em estudos de prevenção de violência pública, visando, como objetivos secundários, pontuar avanços na segurança pública, uma vez que os homicídios se destacam como segunda causa mais comum de morte por causas externas no país (DUARTE EC, et al., 2012).

\section{Local do óbito}

A avaliação epidemiológica do local do óbito é essencial para entender a dinâmica dos homicídios. De modo geral, os estudos mostram maiores taxa de homicídios em áreas de maior vulnerabilidade social. A desigualdade social parece ser um dos argumentos mais comuns para o grande número de homicídios, ainda maior que a pobreza em termos absolutos e está ligada à urbanização e crescimento populacional. Porém, esta variável só foi aprofundada em 2 artigos na revisão bibliográfica realizada para a escrita deste artigo. A falta de aprofundamento dessa variável mostra, novamente, que existe demanda de mais estudos que avaliem os perfis epidemiológicos das vítimas de homicídios, principalmente em nível nacional (GARCÍA HI, et al., 2012; DUARTE EC, et al., 2012).

O artigo da revisão de literatura que mais analisou os detalhes de local do óbito foi o que trata da realização de um estudo ecológico que tinha como objetivo analisar as taxas de homicídio e de lesão corporal ocorridos em Itaboraí, município do interior do Rio de Janeiro dos anos de 2010 a 2011. No trabalho, foram considerados a área total do município, suas áreas críticas e não críticas. $O$ estudo médicolegal associou piores condições de vida ao desfecho de agressões fatais: o homicídio. Foi constatado que em 2011 a taxa de homicídios nas áreas identificadas como críticas foi 53,8\% maior em comparação com a taxa geral de todo o município de Itaboraí. A especulação da relação entre as áreas críticas do município, com os baixos níveis de qualidade de vida e com a violência urbana foi confirmada pelos autores (SOUZA ER, et al., 2018).

Já na África do Sul, as regiões metropolitanas do país apresentaram taxas de mortes maiores em comparação com as regiões não metropolitanas e rurais. Além da diferença da divisão entre as etnias dos homicídios do país, há uma predominância comparativa dos níveis de homicídio como causa de morte por fatores externos em três das nove províncias do país: Eastern Cape, KwaZulu-Natal e Northern Cape (MATZOPOULOS R, et al., 2015).

Em Medellin, na Colômbia, o perfil das vítimas com maior prevalência era dos menores níveis econômicos, residentes das favelas da capital, onde os níveis de escolaridade são baixos, o que pode tornar intrínseca a relação entre pobreza, baixa escolaridade e violência. Isso levou os autores a concluírem que as condições socioeconômicas representam um importante fator de risco para os homicídios. 
trabalho afirma um aumento significativo no número de casos de homicídios ao longo do tempo com uma maior extensão em áreas urbanas, o que tem relação com a urbanização da capital da Colômbia (GARCíA HI, et al., 2012).

\section{Álcool e drogas}

O uso de drogas ilícitas e a alcoolemia foram marcadores comentados por quatro dos artigos analisados nessa revisão sistemática. Dentre eles, uma pesquisa turca deu mais destaque para essa variável de perfil epidemiológico, ao abordar um exame retrospectivo de autopsias de vítimas de mortes por lesões. A pesquisa abordou um exame retrospectivo de autopsias de vítimas de mortes por lesões. $A$ análise dos achados toxicológicos das vítimas de constataram as substâncias mais prevalentes: álcool, com 26,9\% seguido de narcóticos, cannabis e opiáceos (TOYGAR M, et al., 2013).

Os outros artigos analisados pela revisão que abordaram o consumo de álcool ou drogas ilícitas não relataram esses dados com tantos detalhes como a pesquisa feita na Turquia. Em Bogotá há associação entre o consumo de álcool e a ocorrência dos homicídios (GARCíA J, et al., 2018). Outro estudo comenta que a alcoolemia média das vítimas fatais foi de 15,3dg/L nos homens e que nenhuma das vítimas do sexo feminino estava sob influência do álcool (BORDONU PHC, et al., 2017). Enfim, na última pesquisa que abordou o tema aproximadamente um quarto das vítimas analisadas apresentavam resultados positivos na dosagem de alcoolemia, sem diferenças significativas por sexo (EREL Ö, et al., 2011).

É interessante observar a importância do consumo de álcool como variável de perfil epidemiológico de acordo com a cultura. Nos estudos desta revisão com abordagem nesta característica, em diferentes países como a Turquia, foram observadas diferenças nas taxas das vítimas que consumiram álcool e drogas em geral entre os sexos masculino e o feminino (TOYGAR M, et al., 2012; GARCíA HI, et, al, 2012).

\section{Idade e Sexo}

Nessa revisão foi evidente a homogeneidade da classificação das vítimas em relação à idade e ao sexo. Os indivíduos do sexo masculino na faixa etária de 20 a 40 anos predominaram em relação as demais vítimas de homicídios na maioria dos estudos, sendo que indivíduos na faixa de 20 a 30 anos de idade se destacaram em números. De todos os homicídios analisados, mais de 90\% eram homens e menos de $10 \%$ eram mulheres (HE M, et, al., 2015; TAVARES R, et al., 2016; GARCíA, et al., 2012; MATZOPOULOS R, et al., 2015; EREL Ö, et al., 2011; TOYGAR M, et al., 2013).

Em contradição, alguns arquivos trazem dados peculiares que vão além da clássica tendência estatística da mortalidade precoce dos indivíduos do sexo masculino por causas externas, neste caso, por homicídio. Dentre os artigos, destacamos a pesquisa realizada em Xangai, onde houve discrepância estatística em relação aos outros artigos analisados. Na pesquisa chinesa, foram analisados dados de 2696 homicídios ocorridos entre 2000 e 2009 obtendo-se a razão da taxa de homicídios de homens para mulheres de 1,47:1, dado bastante peculiar pela maior taxa de vítimas de homicídio do sexo feminino em comparação aos achados dos demais artigos dessa revisão (HE M, et, al., 2015).

Também foi constatado por essa pesquisa que a idade média das vítimas foi um pouco mais alta do que as faixas predominantes nos outros estudos (de 36,2 $\pm 15,9$ anos). O trabalho também afirma vulnerabilidade de crianças menores de 2 anos a homicídios. Esses dados nos mostram como a diferença de contexto social pode alterar os dados epidemiológicos, bem como marcadores de violência urbana (HE M, et, al., 2015).

\section{Da caracterização das lesões das vítimas de homicídio}

A caracterização das lesões de vítimas de homicídios é um foco deste trabalho, uma vez que está ligado praticamente a todas as palavras-chave usadas ("homicide", "forensics", "autopsy", "weapon" e "injury") e também por ser um tópico importante do ramo da medicina legal. Assim, grande parte dos artigos encontrados na revisão abordaram a caracterização das lesões, sendo alguns com maior riqueza de detalhes do que os outros (HE M, et, al., 2015; TAVARES R, et al., 2016; GARCíA, et al., 2012; MATZOPOULOS R, et al., 2015; EREL Ö, et al., 2011; TOYGAR M, et al., 2013). 
Em relação à caracterização das lesões das vítimas de homicídio, o artigo em que foram avaliados 1888 laudos de homicídios por trauma abdominal, o autor e seus respectivos colaboradores constataram que os óbitos por lesão perfurocontundente foram os de maior acometimento com $78,76 \%$ dos casos, seguidos dos traumas cortante e perfurocortante. Dentre os óbitos por traumas perfurocontundentes, o tipo mais prevalente foi o relacionado a armas de fogo, com $89,28 \%$ dos casos. Nesse estudo, foi comentado que existe diferença na razão entre o sexo masculino e o sexo feminino quando se compara um tipo de lesão à outra. Nos óbitos por trauma perfurocontundente, 93,96\% das vítimas eram homens e 6,4\% eram mulheres. Por fim, quanto aos locais das lesões, foi registrado que os órgãos mais lesados foram fígado, intestinos e baço (BORDONI PHC, et al., 2017).

Em outro estudo que analisa casos de homicídios por trauma de membros, foi observado que as lesões de origem contundente tiveram maior prevalência em relação aos traumas perfurocontundentes por projetil de arma de fogo. Também houve a observação de que, dos 128 casos avaliados com lesões nos membros, 95 indivíduos $(74,2 \%)$ foram identificados com traumas nos membros inferiores, 19 indivíduos $(14,8 \%)$ com traumas nos membros superiores e 13 indivíduos (11\%) com lesões tanto nos membros superiores quanto nos inferiores. Se tratando dos mecanismos de trauma de maneira mais aprofundada, os autores observaram que, na maioria dos casos, ocorreram traumas de origem contundente (49 indivíduos - 38,2\% dos casos), seguidos de traumas de origem perfurocontundente (47 indivíduos - 36,7\% dos casos) e traumas de origem cortante (31 indivíduos - $24,2 \%$ dos casos) (HELENA P, et al., 2017).

Nos homicídios verificados durante o período de estudo em Medellin, na Colômbia, as armas de fogo causaram a maioria dos óbitos. Nesta cidade, $77 \%$ dos casos estudados tiveram como método adotado para execução dos homicídios a utilização de projéteis de arma de fogo (PAF), enquanto menos de $15 \%$ dos óbitos foram atribuídos ao uso de armas perfurocortantes. A maioria das vítimas teve sua morte atribuída a traumas intracranianos e as outras causas foram trauma torácico não especificado, choque hipovolêmico e lesões múltiplas não especificadas (GARCÍA HI, et al., 2012).

Quanto à caracterização das lesões, pouco ainda se encontra na literatura e nesta revisão apenas 1 artigo mostrou essa caracterização a partir de uma pesquisa realizada na África do Sul. Este artigo mostra que dentre as principais causas de homicídio, as de maior frequência são: os traumas por projétil de arma de fogo, os ferimentos cortantes e perfurocortante e, com a menor prevalência, os traumas contundentes. Quanto às mortes que foram causadas por ingestão de substâncias tóxicas, mortes por incêndios e queimaduras por agentes químicos, os autores citam que houve dificuldade na identificação da causa jurídica da morte: se o corrido foi um acidente, um homicídio ou um suicídio. Os autores enfatizam o trauma por projetil de arma de fogo como uma das principais causas de morte por fatores externos na África do Sul, com cerca de 6,5 mil óbitos, dos quais, 85,76\% foram identificadas como homicídios (MATZOPOULOS R, et al., 2015).

No contexto europeu, em uma revisão de casos fatais feita na Polônia, foram examinados 87 casos relacionados a trauma fatal por lesões provocadas por projeteis. O perfil das lesões revelou que os ferimentos pelos projeteis, de modo geral, foram em maioria localizados na cabeça e no peito. Já nos homicídios perpetrados especificamente com o uso de armas de fogo houve uma prevalência de lesões localizadas no tórax (PTASZYŃSKA-SAROSIEK I, et al., 2016).

$\mathrm{Na}$ Turquia, as lesões perfurocontusas por projétil de arma de fogo (PAF) são a principal causa de morte por homicídio. Dentre elas, predomina o disparo a queima roupa, seguido dos disparos encostados na vítima, disparos à distância e os de curta distância. A grande maioria das vítimas morreu com apenas uma lesão por projétil (cerca de 90\%), seguidas numericamente das vítimas que faleceram em decorrência de duas lesões por PAF, e por último, as que evoluíram a óbito com três ou mais lesões por PAF. Na tentativa de descrever o tipo de arma de fogo utilizada nos homicídios, os autores tiveram dificuldades na obtenção desses dados, ficando mais de $40 \%$ dos indivíduos periciados sem confirmação do tipo de arma de fogo que causou sua morte (TOYGAR M, et al., 2013). Tratando-se das regiões anatômicas mais atingidas nos casos de homicídio por arma de fogo, houve destaque na região da cabeça e pescoço, independente da forma como ocorreu a morte a posteriori. Já dentre os orifícios de saída a região mais prevalente foi a região 
cervical, seguida da região torácica posterior com e por último a pelve e extremidades inferiores (TOYGAR M, et al., 2013).

Achados nas necropsias de 1390 corpos em decomposição de vítimas de morte por ação violenta ou suspeita em um estudo realizado pelo Departamento de Medicina Legal da Faculdade de Medicina da Universidade Adnan Menderes nos anos de 2004 a 2008, constataram que os homicídios ocupavam o 4은 lugar no ranking dos tipos de morte dentre as mortes de causa natural, autoextermínio, homicídio e acidentes (EREL Ö, et al., 2011).

\section{CONSIDERAÇÕES FINAIS}

Entender a epidemiologia e os tipos de lesões em homicídios é importante para implementar medidas públicas, além de ser essencial na promoção de saúde da população. A comparação dos dados nacionais com os internacionais pode ser uma referência para a detecção e enfrentamento de questões de segurança pública. Diante disso, é indiscutível a relevância de estudo deste cunho na atual realidade nacional. Os dados desse artigo podem ser relacionados com a atual realidade nacional para a aplicação de medidas públicas, como foi realizada em outros países, o que pode ser uma referência para a diminuição do número de homicídios no Brasil. A diminuição do número de homicídios traz impactos positivos não somente no âmbito econômico, como também nas esferas política e social.

\section{AGRADECIMENTOS}

Ao professor PhD. José Cola Zanuncio, pela revisão, melhorias no texto e metodologia.

\section{REFERÊNCIAS}

1. ANDRADE LH, et al. Mental disorders in megacities: Findings from the São Paulo megacity mental health survey. PLoS One, 2012; 7(2): e31879.

2. ANTÃO KL et al. Perfil epidemiológico de vítimas de violência atendidos em hospital de emergência. Revista Eletrônica Acervo Saúde, 2019; 11(10): e395.

3. BATISTA AS, et al. Metropolização, homicídios e segurança pública na área metropolitana de Brasília: o município de Águas Lindas de Goiás. Soc Estado. 2016; 31(2): 433-57.

4. BARRETO ML, et al. Successes and failures in the control of infectious diseases in Brazil: Social and environmental context, policies, interventions, and research needs. Lancet, 2011; 377(9780), 1877-1889.

5. BORDONI LS, et al. Estudo transversal dos óbitos provocados por trauma nos membros necropsiados no Instituto Médico Legal de Belo Horizonte. Revista Médica de Minas Gerais, 2017; 27: e-1854

6. BORDONI PHC, et al. Óbitos por trauma abdominal: Análise de 1888 autopsias médico-legais. RCLARK C, et al.Patterns of blunt force homicide in the West Metropole of the City of Cape Town, South Africa. South African Journal of Science 2017; 113(5-6): 1-6.

7. DUARTE EC, et al. Associação ecológica entre características dos municípios e o risco de homicídios em homens adultos de 20-39 anos de idade no Brasil, 1999-2010. Ciência \& Saúde Coletiva, 2012; 17(2): 59-68.

8. EREL Ö, et al. Aydin'da bozulmuş cesetlerdeki travmatik bulguları\{dotless\}n değerlendirilmesi. Ulus Travmave Acil CerrahiDerg. 2011; 17(4): 340-3.

9. GARCÍA HI, et al. Treintaaños de homicidiosen Medellín, Colombia, 1979-2008. Cadernos de Saúde Publica. 2012; 28(9): 699-712.

10. GARCÍA J, et al. El feminicidioen Bogotá, una mirada desde elabordaje médico-legal, Cuadernos de Medicina Forense. 2018; 24(2): 27-34.

11. HE M, et al. Unnatural deaths in Shanghai from 2000 to 2009: A retrospective study of forensic autopsy cases at the Shanghai Public Security Bureau. PLoS ONE, 2015; 10(6): 1-10.

12. MATZOPOULOS R, et al. Injury-related mortality in south africa: A retrospective descriptive study of postmortem investigations. Bull World Health Organ, 2015; 93(5): 303-13.

13. MURRAY J, et al. Crime and violence in Brazil: Systematic review of time trends, prevalence rates and risk factors. Aggression and Violent Behavior. 2013: 18(5): 71-83.

14. OLIVEIRA AS. Transição demográfica, transição epidemiológica e envelhecimento populacional no Brasil. Hygeia, 2019; 15(31): $69-79$.

15. ORELLANA JDY, et al. Fatores associados ao homicídio em Manaus, Amazonas, 2014. Epidemiologia e Serviços de Saúde, 2017; 26(4): 735-746.

16. PTASZYŃSKA-SAROSIEK I, et al. Analizaśmiertelnychobrażeńpostrzałowych w materialeZakładuMedycynySądowej w Białymstoku w latach 1964-2015. Archiwum Medycyny Sądoweji Kryminologii. 2016; 66(4): 211-219. 
17. REICHENHEIM ME, et al. Violence and injuries in Brazil: the effect, progress made, and challenges ahead. Lancet 2011; 377: 1962-75.

18. SANTOS JAF, et al. Classe Social, território e desigualdade de saúde no Brasil. Saúde Soc. São Paulo, 2018; 27(2): 556-572

19. DE SOUZA ER, et al. Homicídio e lesão corporal em Itaboraí, Brasil: Análise em diferentes escalas. Ciência e saúde Coletiva. 2018; 23(2): 463-70.

20. TAVARES R, et al. Homicídios e vulnerabilidade social. Ciência e Saúde Coletiva 2016; 21: 923-34.

21. TOYGAR M, et al. An analysis of firearms-related deaths between 1993-2010: a retrospective study. Ulus Travmave Acil CerrahiDerg, 2013; 19(6): 536-42.

22. HAMDOUN M, et al. Patterns of homicide in North Tunisia: a 10-year study (2005-2014). Inj Prev. 2017.

23. WANZINACK C, et al. Homicides and socio-environmental determinants of health in Brazil: a systematic literature review. Cadernos de Saúde Pública 2018. 34(12): 2-21. 